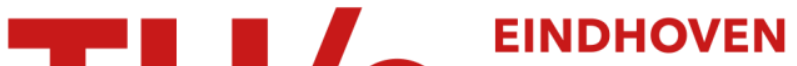

\section{Frequency stabilization of an InP-based integrated diode laser deploying electro-optic tuning}

Citation for published version (APA):

Andreou, S., Williams, K., \& Bente, E. (2019). Frequency stabilization of an InP-based integrated diode laser deploying electro-optic tuning. IEEE Photonics Technology Letters, 31(24), 1983-1986. [8894117].

https://doi.org/10.1109/LPT.2019.2952243

DOI:

10.1109/LPT.2019.2952243

Document status and date:

Published: 15/12/2019

Document Version:

Accepted manuscript including changes made at the peer-review stage

Please check the document version of this publication:

- A submitted manuscript is the version of the article upon submission and before peer-review. There can be important differences between the submitted version and the official published version of record. People interested in the research are advised to contact the author for the final version of the publication, or visit the $\mathrm{DOI}$ to the publisher's website.

- The final author version and the galley proof are versions of the publication after peer review.

- The final published version features the final layout of the paper including the volume, issue and page numbers.

Link to publication

\section{General rights}

Copyright and moral rights for the publications made accessible in the public portal are retained by the authors and/or other copyright owners and it is a condition of accessing publications that users recognise and abide by the legal requirements associated with these rights.

- Users may download and print one copy of any publication from the public portal for the purpose of private study or research.

- You may not further distribute the material or use it for any profit-making activity or commercial gain

- You may freely distribute the URL identifying the publication in the public portal.

If the publication is distributed under the terms of Article 25fa of the Dutch Copyright Act, indicated by the "Taverne" license above, please follow below link for the End User Agreement:

www.tue.nl/taverne

Take down policy

If you believe that this document breaches copyright please contact us at:

openaccess@tue.nl

providing details and we will investigate your claim. 


\title{
Frequency stabilization of an InP-based integrated diode laser deploying electro-optic tuning
}

\author{
Stefanos Andreou, Kevin A. Williams, Erwin A. J. M. Bente
}

\begin{abstract}
We present the frequency stabilization of a monolithically integrated extended cavity single mode InP diode laser using the Pound-Drever-Hall (PDH) frequency locking technique. The laser is a multi-section distributed Bragg reflector (DBR) laser with an intra-cavity ring resonator, fabricated using an InP active-passive integration technology. The laser is locked to a $700 \mathrm{kHz}$ wide resonance of a Fabry-Perot etalon. The single electrical feedback is applied on the reverse biased rear DBR section of the laser, used to tune the lasing mode. This is the first time to our knowledge that the feedback is applied on a reverse biased, voltage controlled section of an integrated laser cavity. In our implementation the tuning is based on electro-optic effects avoiding significant thermal effects in the tuning element. We demonstrate a linewidth reduction down to $5 \mathrm{kHz}$ and frequency noise suppression of about $30 \mathrm{~dB}$ at $10 \mathrm{~Hz}$ offset frequency. The bandwidth of the control loop is about $500 \mathrm{kHz}$, limited by the phase delay of components in our loop.
\end{abstract}

Index Terms - Pound-Drever-Hall locking, laser stabilization, InP laser, electro-refractive modulator, linewidth

\section{INTRODUCTION}

$\mathrm{L}$ ASERS with good short- and long-term stability are Uessential in high-resolution sensing, spectroscopy and metrology. The stability of free-running lasers is greatly compromised by environmental disturbances such as temperature and pressure fluctuations, vibrations and flicker noise from electronics. Often, depending on the application, their intrinsic linewidth [1] is not sufficiently low either. In this case stabilization schemes are required to dramatically improve the stability of free-running lasers. Such schemes may deploy electrical or optical feedback, feedforward techniques or even combinations of these.

The most powerful and widely used technique is the PDH frequency locking [2]. In PDH locking, the laser is usually locked to the resonance of a high-finesse optical cavity acting as an absolute reference. If the electrical feedback loop has sufficient bandwidth and correct gain it can not only correct for frequency drifts but also reduce the linewidth of a laser by

This work was supported by the Netherlands organization for scientific research (NWO) under the project 13930 and the United States Air Force Office of Scientific Research (AFOSR) under the grant FA9550-18-1-0015. suppressing the phase noise resulting from both technical noise and amplified spontaneous emission (ASE) within this bandwidth. The feedback is usually applied on the semiconductor optical amplifier (SOA) section [3] of the laser or current injection phase sections in the cavity [4].

The combination of current-induced thermo-optic tuning in the millisecond time scale and the carrier density variation (plasma effect) in the nanosecond scale causes a non-flat laser tuning phase response, typically above $100-200 \mathrm{kHz}[5,6]$. The two effects also result in opposite frequency shifts with increasing current. All of the above many times lead to the use of two feedback loops at different frequency ranges or the design of special feedback loops as proposed in [3] to circumvent the non-flat laser tuning phase response. Further issues, which however are not investigated here, can arise when feedback is applied in the form of current-injection such as excess intensity noise which may limit the frequency noise suppression $[3,5]$. Additionally, the laser tuning response is dependent on the SOA current. This means that if the SOA current significantly changes over time due to a large drift, the feedback loop may no longer be optimized for the new conditions [5]. Finally, depending on the use of the stabilized laser, one may want to avoid additional intensity noise. For example as explained in [7] where an extended cavity diode laser in Littman configuration is used, the feedback is preferably applied on an intra-cavity lithium niobate modulator instead of the SOA.

In this work, we investigate and demonstrate the stabilization of an InP-based diode laser using PDH locking by applying the electrical feedback on the reverse biased DBR section of the laser to tune the lasing mode. The reverse bias tuning implementation is based on electro-optic effects and avoids
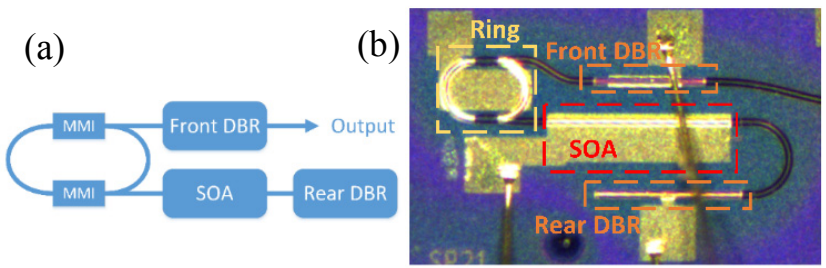

Fig. 1. (a) Schematic and (b) image of the integrated InP-based DBR laser with an intra-cavity ring resonator. SOA: semiconductor optical amplifier, DBR: distributed Bragg reflector, MMI: multimode interference coupler.

S. Andreou, Kevin A. Williams and E. A. J. M. Bente are with the Photonic Integration group, Department of Electrical Engineering, Eindhoven University of Technology, Eindhoven, The Netherlands (e-mail: s.andreou@tue.nl). 
significant thermal effects contrary to feedback with current injection. The bandwidth of the tuning mechanisms does not limit the control loop bandwidth in our case since the response time is on the nanosecond timescale [8]. In section II the laser cavity is described and the implementation of the PDH locking to a highly-stable Fabry-Perot cavity are given. In section III the stabilization is presented.

\section{IMPLEMENTATION OF THE PDH LOCKING WITH AN INP-BASED LASER}

A schematic and an image of the InP-based laser fabricated in the framework of a multi-project wafer run using an activepassive integration technology [8] is shown in Fig. 1(a) and (b) respectively. It is a multi-section DBR laser with an intra-cavity ring resonator [9]. The SOA is $500 \mu \mathrm{m}$ long and the front and rear DBRs are $300 \mu \mathrm{m}$ and $400 \mu \mathrm{m}$ long respectively with coupling coefficient of $50 \mathrm{~cm}^{-1}$. The ring resonator has an $80 \mu \mathrm{m}$ radius and power coupling is implemented using 50:50 $2 \times 1$ multimode interference couplers (MMI).

The DBR sections have the same cross-section (disregarding the local differences in the grating layer for the weak effective index modulation) as the electro-refractive phase modulators in the same technology. They can be used in reverse or forward bias to change the effective index. The propagating mode is guided in a film of the bulk quaternary material with bandgap at $1.25 \mu \mathrm{m}$ [6]. Typically reverse bias voltages up to $10 \mathrm{~V}$ are applied. As we have shown in [10], the reverse bias tuning does not cause significant losses. Additional losses $(<2 \mathrm{~dB} / \mathrm{cm})$ are expected only at voltages from -5 to $-10 \mathrm{~V}$ and slight reduction $(0.5 \mathrm{~dB} / \mathrm{cm})$ of losses from 0 to $-5 \mathrm{~V}$. Therefore maximum additional losses may be roughly $0.08 \mathrm{~dB}(0.04 \mathrm{~cm} \times 2 \mathrm{~dB} /$ $\mathrm{cm}$ ). These losses are significantly lower than the total intracavity loss which is estimated to be $4-5 \mathrm{~dB}$. The laser threshold current is not expected to vary significantly. In reverse bias, the change of the effective index of the guided mode results from primarily electro-optic-effects $[10,11]$, the Pockels and Kerr effects. Weaker carrier effects are also present, free-carrier plasma and band-filling effects. These are typically an order of magnitude smaller compared to field effects due to low doping levels. Since the current levels flowing through the PIN junction in reverse bias operation are in the order of some $\mu \mathrm{A}$, thermo-optic tuning is expected to be negligible [12]. This is in contrast with the electric feedback applied on current injection phase sections and/or the laser SOA. In both cases the thermal dissipation is significant. Additionally the thermal tuning has the opposite sign compared to carrier injection tuning therefore two different control signals are typically used.

The integrated laser was fabricated by Smart Photonics [8] The chip is mounted on an aluminum sub-mount and kept at $18^{\circ} \mathrm{C}$. Electrical signals are applied using wire-bonds from a printed circuit board to the on-chip bond-pads. The SOA is driven at $\sim 60 \mathrm{~mA}$ using a low-noise battery based laser diode controller (ILX LDX-3620B). Light from the laser is coupled out of the chip using a single-mode lensed fibre. The output waveguides are angled with respect to the chip facet which is coated in order to avoid back-reflections into the laser cavity. A

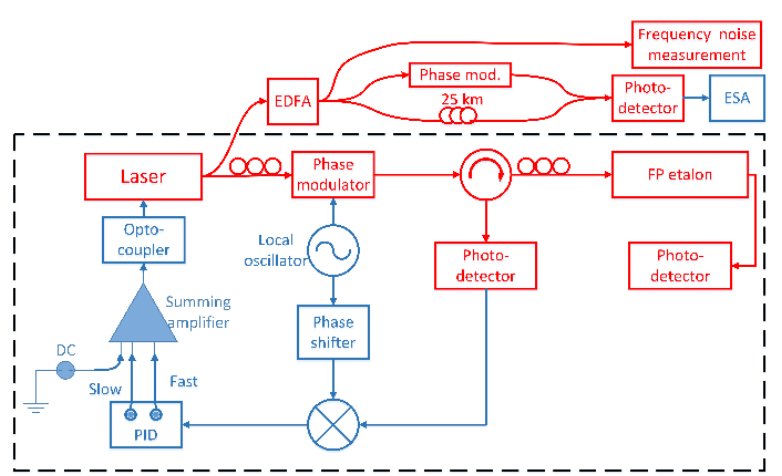

Fig. 2. Schematic of the Pound-Drever-Hall frequency locking used for the diode laser stabilization.

schematic of the experimental setup used is depicted in Fig. 2. The laser output is phase modulated at $60 \mathrm{MHz}$ using a lithium niobate modulator. The modulated light is fed to the FabryPerot cavity through a circulator. The cavity has a full-width at half-maximum (FWHM) of $\sim 700 \mathrm{kHz}$ (StableLasers). Its finesse is $4.5 \cdot 10^{3}$ and it is made out of ultra-low expansion material. The signal which is reflected from the cavity is directed to a low-noise photodetector (PD, Femto OE-300-IN$01)$ with a variable transimpedance gain (transimpedance gain: $10^{4} \mathrm{~V} / \mathrm{A}$, bandwidth: $80 \mathrm{MHz}$ ). It is down-converted to baseband and fed to the analogue PID controller with two outputs, high- and low-bandwidth. The $60 \mathrm{MHz}$ oscillator, mixer, phase shifter and analogue PID controller are commercial locking electronics from Toptica (PDD110, FALC110).

Between the PID controller and the integrated laser we place an opto-coupler to electrically isolate the laser and avoid ground loops which leads to spurious signals. The opto-coupler is realized using a visible light emitting diode (LED) and a battery-biased large area PD. A potentiometer with maximum resistance value of $1 \mathrm{k} \Omega$ is connected in series with the largearea PD and the voltage drop across the potentiometer is the output voltage of the opto-coupler. We estimate that for the maximum resistance value of the potentiometer the lowest bandwidth is $0.5 \mathrm{MHz}$. The PID controller has two outputs, a slow output with cut-off frequency of $10 \mathrm{kHz}$ and a fast output with a two stage integrator (cut-off frequencies: $14 \mathrm{kHz}$ and $370 \mathrm{kHz}$ ) and a differentiator (cut-off frequency: $420 \mathrm{kHz}$ ). The two outputs are summed with a DC voltage using a summing
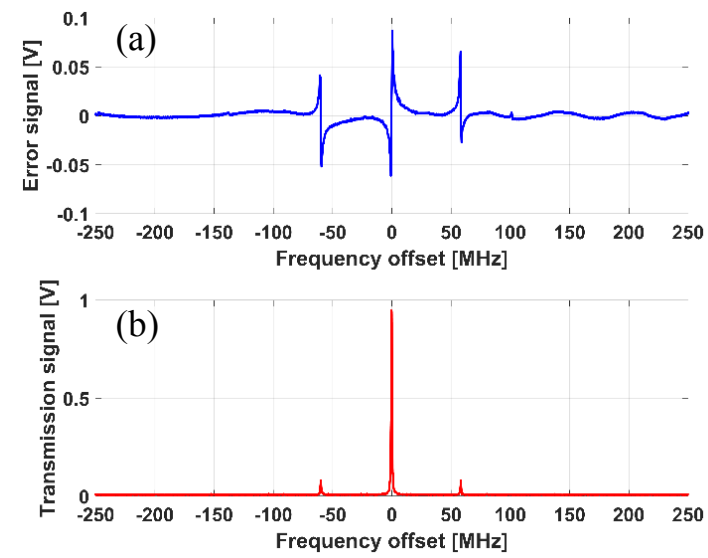

Fig. 3. (a) The error (blue) and (b) transmission (red) signal through the FabryPerot etalon versus the offset frequency from a resonance of the cavity. 
amplifier (Stanford Research SIM980) with $1 \mathrm{MHz}$ bandwidth. The summing amplifier output biases the LED of the optocoupler. The output voltage of the opto-coupler is then applied to the rear DBR section of the laser.

We split the light with a fibre-based splitter and we use $10 \%$ of the laser output power to measure its linewidth and frequency noise while the remaining signal is used for the locking. The linewidth is measured using the delayed self-heterodyne (DSH) [13] method with a delay line of $25 \mathrm{~km}$ and an electrical spectrum analyzer (ESA - Anritsu MS2830A). The frequency noise is measured using a frequency noise measurement instrument from OEwaves (OE4000). For both measurements, we amplify the optical signal using a low-noise figure $(4 \mathrm{~dB})$ erbium doped fibre amplifier (EDFA - Keopsys CEFA-C-HGSM-50-B130) in order to have sufficient optical power $(>5 \mathrm{dBm})$. At the output of the EDFA a narrow-band bandpass filter $(0.5 \mathrm{~nm})$ is placed to filter out the ASE.

\section{LOCKING MEASUREMENTS}

The open loop error signal as measured at the output of the PID controller is shown in Fig. 3(a) (blue trace) as a function of the detuning frequency of the laser from the resonance transmission maximum. The resulting slope of the error signal around zero frequency offset is $190 \mu \mathrm{V} / \mathrm{kHz}$. The sensitivity of the laser frequency control can be determined from the same trace in Fig. 3(a). The zero-crossing points left and right are $60 \mathrm{MHz}$ away from the centre. Using this information the sensitivity of the laser frequency control is determined to be $320 \mathrm{kHz} / \mathrm{mV}$. Therefore given the $10 \mathrm{~V}$ biasing range, the frequency control range is about $3.2 \mathrm{GHz}$. The small ripples which are visible in the error signal correspond to the $50 \mathrm{~Hz}$ frequency from the equipment power supply. The transmission (red) through the FP cavity is shown in Fig. 3(b). The two smaller peaks correspond to the phase modulation side-bands at $60 \mathrm{MHz}$.

In Fig. 4(a) the linewidth of both free-running (blue line) and locked laser (orange line) were recorded using an ESA with a resolution bandwidth of $10 \mathrm{kHz}$ and video bandwidth of $1 \mathrm{kHz}$ using the DSH method. The linewidth of the laser is reduced and the peak power density increased. The free-running laser exhibits a Gaussian linewidth, dominated by technical noise. The lineshape of the locked laser shows sub-structure. The peaks that appear at about $800 \mathrm{kHz}$ offset are due to the bandwidth limitation of our feedback loop. At higher frequency offsets the lineshape tails follow the lineshape of the freerunning laser. In Fig. 4(b) the electrical spectra are shown with resolution $\mathrm{BW}$ of $1 \mathrm{kHz}$ and video $\mathrm{BW}$ of $100 \mathrm{~Hz}$. For this span the lineshape is Lorentzian. A single sweep of the ESA recording the linewidth is presented in light orange and an average of twenty sweeps with darker orange. The FWHM of the locked laser is $5 \mathrm{kHz}$. This is a factor of more than 100 lower that the reference cavity resonance FWHM. The free-running laser (blue line) shows a linewidth of about $300 \mathrm{kHz}$. Therefore the locking system reduces the linewidth by a factor of about 60 .

In Fig. 5 the power spectral density (PSD) of the free-running (blue line) and frequency-locked laser (orange line) frequency
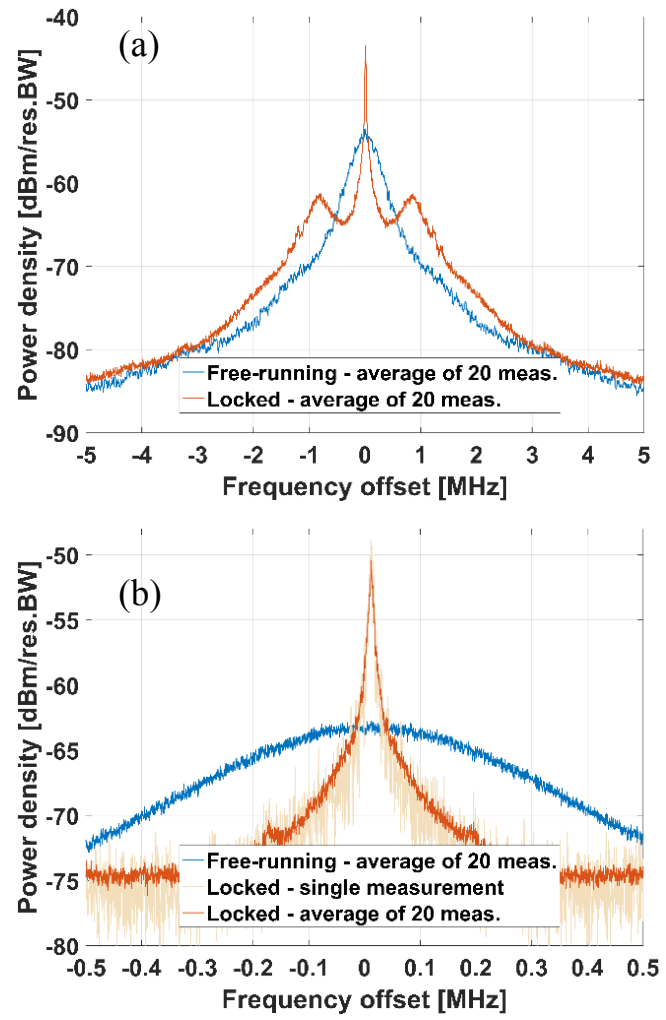

Fig. 4 Electrical spectra from the delayed self-heterodyne setup with $25 \mathrm{~km}$ delay line with (a) $10 \mathrm{MHz}$ and (b) $1 \mathrm{MHz}$ span. The linewidth is reduced from $\sim 300 \mathrm{kHz}$ for the free-running laser to about $5 \mathrm{kHz}$.

noise is presented. The free-running laser frequency noise exhibits $1 / \mathrm{f}^{\alpha}$ noise, where $0<\alpha<2$, with varying slope from $10 \mathrm{~Hz}$ to about $1 \mathrm{MHz}$. Multiple spurious peaks are present with the most prominent line at $50 \mathrm{~Hz}$ and its harmonics. Additionally a strong peak at $\sim 0.9 \mathrm{kHz}$ is observed. The origin of these peaks is possibly electrical pick-up signals and/or mechanical vibrations of the fibre tip used for coupling light out of the chip.

By comparing the frequency noise for the free-running and locked lasers we observe noise suppression by the control loop of almost $30 \mathrm{~dB}$ for the lowest frequencies. The gain reduces gradually until it reaches unity at $500 \mathrm{kHz}$. In the whole range multiple spurious signals are present. The origin of these spurious signals is not known at the moment. The wide peak which is located at $\sim 800 \mathrm{kHz}$ is a result of the phase lag of our feedback loop and corresponds to the same peaks exhibited in the DSH measurements (Fig. 4). At these frequencies the feedback is no longer effective due to the phase lag caused by both the round-trip time delay and the frequency dependent phase delay from components included in the loop. As discussed in section II, the opto-coupler bandwidth is expected to be about $\sim 0.5 \mathrm{MHz}$ therefore being the limiting factor for the loop bandwidth.

\section{DISCUSSION}

In Fig. 5 the $\beta$-separation line expressed by $\beta_{\text {sep }}(f)=$ $8 \ln (2) f / \pi^{2}[14]$ is plotted which offers a straightforward link between the frequency noise spectrum and linewidth. The part of the frequency noise that contributes to the linewidth as 


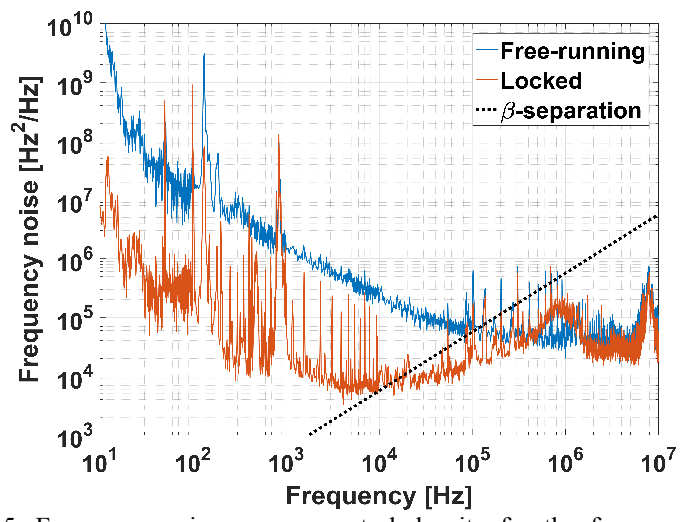

Fig. 5. Frequency noise power spectral density for the free-running and locked laser. The frequency noise at low frequencies is suppressed by about $30 \mathrm{~dB}$ and the control loop bandwidth is about $500 \mathrm{kHz}$.

measured with the DSH method is the part for which $S_{v}(f)>$ $\beta_{\text {sep }}(f)$, where $S_{v}(f)$ is the laser frequency noise spectrum. The expression for the laser FWHM is $\Delta v=(8 \cdot \ln (2) \cdot A)^{0.5}$ [10] where $A$ is the surface area where $S_{v}(f)>\beta_{\text {sep }}(f)$. This can be calculated from the measured $S_{v}(f)$ and it depends on the observation time - the frequency range over which $S_{v}(f)$ is integrated.

From Fig. 5, we find that for the free-running laser, frequency noise below $100 \mathrm{kHz}$ contributes to the linewidth while for the locked laser this frequency decreases to about $10 \mathrm{kHz}$. Since our control loop bandwidth is about $500 \mathrm{kHz}$, which is greater than $100 \mathrm{kHz}$, to further reduce the linewidth we do not need a control loop with higher bandwidth. Further linewidth reduction requires higher gain at frequencies below $10 \mathrm{kHz}$. An integrator with higher gain at these low frequencies could be deployed to achieve this.

To check the consistency of our DSH measurements with the frequency noise measurements we numerically integrate the surface for which $S_{v}(f)>\beta_{\text {sep }}(f)$. The $25 \mathrm{~km}$ delay used in our DSH setup corresponds to a delay/observation time of approximately $120 \mu \mathrm{s}$. For the free-running laser we integrated the frequency noise spectrum from $8 \mathrm{kHz}$ to $100 \mathrm{kHz}$ resulting in a linewidth of $280 \mathrm{kHz}$. For the locked laser the noise spectrum was integrated from $8 \mathrm{kHz}$ to $10 \mathrm{kHz}$ resulting in an $8 \mathrm{kHz}$ linewidth. These numbers are reasonably close to our observations from the electrical spectra (Fig. 3). The spurious peaks in the frequency noise spectrum slightly contribute to the overestimation of the linewidth calculated for the locked laser.

According to our calculations the stabilization system is not limited by the signal-to-noise ratio (SNR) at the PD. Further suppression of the noise by at least an order of magnitude should be possible based only on the SNR at the PD. We attribute the current gain limitations of our stabilization system to the spurious peaks in the frequency noise spectrum.

\section{CONCLUSIONS}

We demonstrate the PDH locking of a monolithically integrated extended cavity single frequency laser using a single feedback loop utilizing electro-optic effects. The electrical feedback is applied on the reverse biased rear DBR section of the laser yielding a frequency control range of about $3.2 \mathrm{GHz}$. The electro-optic effects deployed for the electrical feedback due to the reverse bias operation all have identical signs and avoid significant heat dissipation. This tuning mechanism avoids nonflat laser tuning phase response and enable the utilization of a single feedback loop without special design compared to feedback using current injection on phase sections or SOAs. The control loop bandwidth is $500 \mathrm{kHz}$ limited by the bandwidth of the opto-coupler used. We measure linewidth reduction of a factor of 60 compared to the free-running laser and about a factor of 140 of the etalon FWHM. The frequency noise measurements show that the control bandwidth is sufficient to suppress high-frequency noise components in order not to contribute to the linewidth. Further linewidth reduction requires higher gain in the lower frequencies. This method is promising for overcoming other shortcomings of current injection feedback such as intensity noise as well.

\section{REFERENCES}

[1] A. L. Schawlow and C. H. Townes, "Infrared and optical masers," Physical Review, vol. 112, no 16, pp. 1940-1949, Dec. 1958.

[2] R. W. P. Drever et al., "Laser phase and frequency stabilization using an optical resonator," Applied Physics B, vol. 31, no 12, pp. 97-105. 1983.

[3] D.T. Spencer, M.L. Davenport, T. Komljenovic, S. Srinivasan and J.E. Bowers, "Stabilization of heterogeneous silicon lasers using PoundDrever-Hall locking to Si3N4 ring resonators," Opt. Express, vol. 24, no. 12, pp. 13511-13517, 2016.

[4] A. Sivananthan et al., "Monolithic linewidth narrowing of a tunable SGDBR laser," 2013 Optical Fiber Communication Conference and Exposition and the National Fiber Optic Engineers Conference (OFC/NFOEC), Anaheim, CA, 2013, pp. 1-3, OTh3I.3.

[5] M. Ohtsu and N. Tabuchi, "Electrical Feedback and its Network Analysis for Linewidth Reduction of a Semiconductor Laser," J. Light. Technol., vol. 6, no. 3, pp. 357-369, 1988.

[6] J.L. Hall, M.S. Taubman, and J. Ye, "Frequency stabilization" in Handbook of Optics, $3^{\text {rd }}$ edition, vol. II. M. Bass et al., New York: The McGraw-Hill Companies, 2009.

[7] H. Stoehr, F. Mensing, J. Helmcke, and U. Sterr, "Diode laser with $1 \mathrm{~Hz}$ linewidth," Opt. Lett., vol. 31, no. 6, pp. 736-738, 2006.

[8] L. M. Augustin et al., "InP-Based Generic Foundry Platform for Photonic Integrated Circuits," IEEE Journal of Selected Topics in Quantum Electronics, vol. 24, no 11, pp. 1-10, 2018.

[9] S. Andreou, K.A. Williams, and E.A.J.M. Bente, "Monolithically integrated InP-based DBR lasers with an intra-cavity ring resonator," Opt. Express, vol. 27, no. 19, pp. 26281-26294, 2019.

[10] S. Andreou, K. A. Williams and E. A. J. M. Bente, "Steady-State Analysis of the Effects of Residual Amplitude Modulation of InP-Based Integrated Phase Modulators in Pound-Drever-Hall Frequency Stabilization," in IEEE Photonics Journal, vol. 11, no. 3, pp. 1-14, 2019.

[11] W. Yao, "Towards a High-Capacity Multi-Channel Transmitter in Generic Photonic Integration Technology," Ph.D. dissertation, Dept. Elect. Eng. Eindhoven Univ. of Technology, Eindhoven, The Netherlands, 2017.

[12] R. Pajković, Y. Tian, S. Latkowski, K. Williams, E. Bente, "Tuning of a widely tunable monolithically integrated InP laser for optical coherence tomography," Proc. SPIE 10939, Novel In-Plane Semiconductor Lasers XVIII, 1093912, 2019.

[13] T. Okoshi, K. Kikuchi and A. Nakayama, "Novel method for high resolution measurement of laser output spectrum," in Electronics Letters, vol. 16, no. 16, pp. 630-631, 1980.

[14] G. Di Domenico, S. Schilt, and P. Thomann, "Simple approach to the relation between laser frequency noise and laser line shape," Appl. Opt., vol. 49 , no. 25 , pp. 4801-4807, 2010. 